\title{
Correlation of PK/PD Indices with Resistance Selection for Cefquinome against Staphylococcus aureus in an In Vitro Model
}

\author{
Yafei Lit', Baoyi Feng ${ }^{1+}$, Xiaoyan $\mathrm{Gu}^{2}$, Dawei Yang ${ }^{3}$, Zhenling Zeng ${ }^{1}$, Bingxu Zhang ${ }^{1}$ and \\ Huanzhong Ding ${ }^{1 *}$ \\ 'Laboratory of Veterinary Pharmacology, College of Veterinary Medicine, South China Agricultural University, Guangzhou, \\ China, ${ }^{2}$ Centre for Veterinary Drug Residues, College of Veterinary Medicine, South China Agricultural University \\ Guangzhou, China, ${ }^{3}$ China Institute of Veterinary Drug Control, Beijing, China
}

OPEN ACCESS

Edited by:

Alexandre Gonçalves, Universidade de Trás-os-Montes e Alto Douro, Portugal

Reviewed by:

Dmitri Debabov,

NovaBay Pharmaceuticals, Inc., USA

William Farias Porto,

Universidade Católica de Brasília,

Brazil

*Correspondence:

Huanzhong Ding

hzding@scau.edu.cn

${ }^{\dagger}$ These authors have contributed equally to this work.

Specialty section:

This article was submitted to Antimicrobials, Resistance

and Chemotherapy

a section of the journal

Frontiers in Microbiology

Received: 30 August 2015 Accepted: 21 March 2016

Published: 12 April 2016

Citation:

$L i Y$, Feng $B$, Gu $X$, Yang $D$, Zeng $Z$,

Zhang $B$ and Ding $H$ (2016)

Correlation of PKJPD Indices with

Resistance Selection for Cefquinome

against Staphylococcus aureus

in an In Vitro Model.

Front. Microbiol. 7:466.

doi: 10.3389/fmicb.2016.00466
Cefquinome is a fourth-generation Cephalosporin approved for use in animals exclusively. The objective of this study was to explore the relationship of cefquinome pharmacokinetic/pharmacodynamic (PK/PD) indices with resistance selection of Staphylococcus aureus ATCC25923 in an in vitro model. Six dosing regiments of cefquinome at an interval of $24 \mathrm{~h}$ for three consecutive times were simulated, resulting in maximum concentrations $\left(\mathrm{C}_{\max }\right)$ from $1 / 2$ to $16 \mathrm{MIC}$ and terminal half-lives $\left(\mathrm{t}_{1 / 2 \beta}\right)$ of 3 and $6 \mathrm{~h}$, respectively. The in vitro sensitivity of $S$. aureus was monitored by bacterial susceptibility and dynamic time-kill curve experiments over the six cefquinome concentrations. The correlation between changes in bacterial susceptibility $\left(\mathrm{MIC}_{72} / \mathrm{MIC}_{0}\right)$ and the percentage of time within mutant selection window versus dosing interval ( $\left.T_{M S W} \%\right)$ was subjected to the Gaussian function and regression analysis. Our results favored the consensus that time above $\mathrm{MIC}(\mathrm{T}>\mathrm{MIC})$ was recognized as an important PK/PD parameter of cephalosporins for antibacterial efficiency. Cefquinome reached the maximum killing effect when T > MIC\% attained approximately 40 60\%. The subsequent correlation analysis demonstrated that resistant S. aureus ATCC25923 was easy to occur when $T_{M S W} \%$ attained an index of about $20 \%$ with $t_{1 / 2 \beta}$ of $3 \mathrm{~h}$ after multiple dosing, and $40 \%$ with $t_{1 / 2 \beta}$ of $6 \mathrm{~h}$ after multiple dosing, respectively.

Keywords: cefquinome, Staphylococcus aureus, PK/PD, MPC, in vitro model

\section{INTRODUCTION}

Staphylococcus aureus is a major pathogen for animals and humans, which contributes to a variety of severe infections, including bacteremia, meningitis, endocarditis, skin and wood infection or other diseases for animals and humans (Archer, 1998; Lowy, 1998; Azizoglu et al., 2013). More importantly, S. aureus plays an important role in the food contamination by foodborne pathogens. Food poisoning caused by staphylococcal enterotoxin (SE) is a pressing worldwide health problem (Tauxe, 2002; Le Loir et al., 2003). In spite of the progress in antimicrobial therapy, treatment of $S$. aureus infection has become more and more challenging 
because drug-resistance of $S$. aureus has increased globally over the past decade. The methicillin-resistant S. aureus (MRSA) and vancomycin-resistant $S$. aureus (VRSA) were particularly reported and well documented (Harris et al., 2010; Mohammed Fayaz et al., 2011).

In veterinary medicine, cefquinome (CEQ) has been licensed in European countries by virtue of its broad antibacterial spectrums and remarkable antibacterial activities. Despite of this, the cephalosporins should be in prudent use considering the escalating antimicrobial resistance. Evidence has showed that different animal species may harbor the same resistance determinant and are recognized as possible reservoirs of antimicrobial-resistant bacteria (Guardabassi et al., 2004). It has been also observed that cefquinome exerted a selective effect

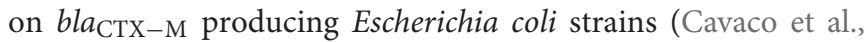
2008). Therefore, further information concerning the ability of cefquinome to prevent occurring of resistant strains seems to be investigated.

Since mutant prevention concentration (MPC) of antibiotics was first described by Dong et al. (1999), it has been successfully applied to evaluate the ability of antibiotics that restrict the selection of resistant strains (Allen et al., 2004; Allen and Hankins, 2009; Wang et al., 2010; Blondeau et al., 2012) and optimize the current dosing regimens of antibiotics to slow the emergence of resistant strains. MPC was taken as the lowest doubling dilution drug concentration that prevented a population of $10^{10}$ colony forming unit (CFU)/mL or even more microorganisms from first-step mutation. Zone of the drug concentrations between minimum inhibitory concentration (MIC) and MPC was defined as the mutant selection window (MSW). MPC and MSW concepts, representing the ability of antibiotics to select resistant strains, have been tested in various in vitro studies(Allen et al., 2004; Croisier et al., 2004b; Ferran et al., 2007), in ex vivo pharmacodynamic studies (Bronner et al., 2002) and in in vivo studies (Croisier et al., 2004a; Cui et al., 2006). Here, we developed an in vitro kinetic model to investigate the relationship between pharmacokinetic/pharmacodynamic $(\mathrm{PK} / \mathrm{PD})$ indices of cefquinome with terminal half-lives $\left(\mathrm{t}_{1 / 2 \beta}\right)$ of 3 or $6 \mathrm{~h}$ and resistance development of S. aureus ATCC25923.

\section{MATERIALS AND METHODS}

\section{Antimicrobial Agents, Medium, and Bacterial Strains}

Raw material of cefquinome (purity of $84.1 \%$ ) was obtained from Hebei yuanzheng Pharmaceutical Enterprise, Co., Ltd. MuellerHinton broth (MHB) and agar were purchased from Guangzhou huankai Comapany. S. aureus ATCC 25923 was purchased from the China Institute of Veterinary Drug Control (Beijing, China).

\section{In Vitro Susceptibility Testing}

The MIC of CEQ against $S$. aureus with an inoculum of $5 \times 10^{5}$ $\mathrm{CFU} / \mathrm{mL}$ was determined by standard agar dilution method established by the Clinical and Laboratory Standards Institute (CLSI).

\section{Measurement of MIC $_{99}$, Mutant Prevention Concentration (MPC) and Selection Index (SI)}

The $\mathrm{MIC}_{99}$ was defined as the drug concentration that inhibited $99 \%$ of bacteria colony formation. MPC was reckoned as the lowest cefquinome concentration blocking $\geq 10^{10} \mathrm{CFU} / \mathrm{mL}$ inoculants growth. The measurements of $\mathrm{MIC}_{99}$ and MPC were mainly based on the method reported by Zhao and Drlica (2002) with slight modification. For MIC99, agar plates containing a series of cefquinome (concentrations ranging from 0.5 to $0.164 \mu \mathrm{g} / \mathrm{mL}$ ) at $20 \%$ per sequential decrease were prepared. $3 \times 10^{7} \mathrm{CFU} / \mathrm{mL}$ S. aureus suspension in logarithmic phase of growth was then subjected to serial 10 -fold dilutions with $\mathrm{MHB}$ to $3 \times 10^{2} \mathrm{CFU} / \mathrm{mL}$ bacteria. Again, $100 \mu \mathrm{L}$ of each dilution was plated onto the agar plates containing the series of CEQ concentrations mentioned above and incubated at $37^{\circ} \mathrm{C}$ overnight. Bacterial colonies that recovered growth in each dilution were counted. Drug concentrations versus the percentages of colony recovery were plotted and the interpolation method was adopted to calculate the cefquinome concentration blocking 99\% bacteria growth.

The determination of MPC was similar to that of $\mathrm{MIC}_{99}$. Instead, $100 \mu \mathrm{L}$ of $10^{10} \mathrm{CFU} / \mathrm{mL}$ inoculants was spread on agar plates containing a series of antimicrobial concentrations. The lowest antimicrobial concentration preventing bacterial colony formation at $72 \mathrm{~h}$ after incubation was measured as provisional mutant prevention concentration $\left(\mathrm{MPC}_{\mathrm{pr}}\right)$. A second determination that utilized linear $\mathrm{MPC}_{\mathrm{pr}}$ decreases (about 20\% per sequential decrease) was performed. MPC was the lowest CEQ concentration preventing $100 \%$ growth of S. aureus colony.

The calculated ratio of $\mathrm{MPC} / \mathrm{MIC}_{99}$ for $S$. aureus was defined as selection index (SI) of cefquinome.

\section{In Vitro Dynamic PK/PD Model Simulation}

A previous described dynamic model (Grasso et al., 1978) with modification was developed in this study. One-compartment open model with first-order absorption of pharmacokinetics for cefquinome was simulated. The schematic representation of in vitro $\mathrm{PK} / \mathrm{PD}$ model used is depicted in Figure 1. Briefly, the system was mainly composed of three sealed containers

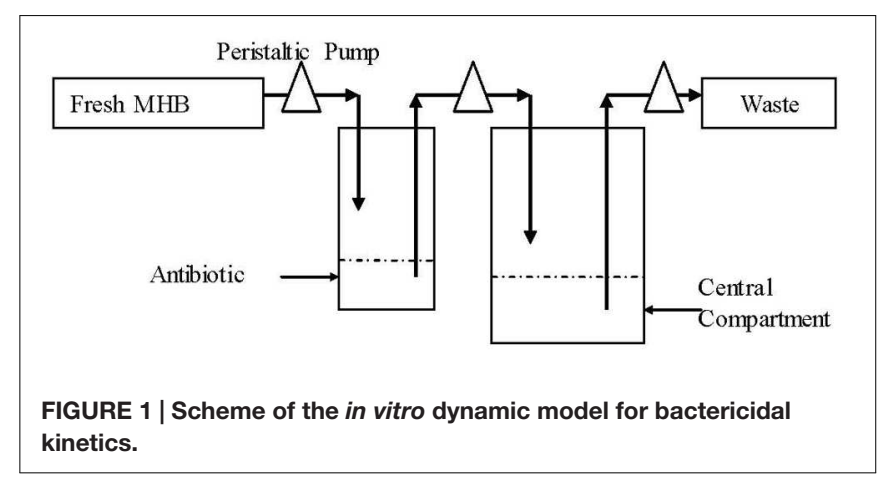


(compartments) and they were connected with peristaltic pumps in line, each containing sterile $\mathrm{MHB}$ and a magnetic stirrer to ensure adequate mixing. One $500 \mathrm{~mL}$ sealed compartment containing $300 \mathrm{~mL} \mathrm{MHB}$, provided with either a bacterial culture alone (control growth experiments) or a bacterial culture plus antibiotic (killing/re-growth experiments), acted as the central chamber. Another $100 \mathrm{~mL}$ sealed container charged with $60 \mathrm{~mL}$ of sterile $\mathrm{MHB}$, acted as the absorption chamber, with desired calculated drug concentrations. The third one was used to provide fresh MHB. Waste was also collected. Peristaltic pumps circulated fresh broth to central and absorption compartments at the desired flow rate. The flow rate of the pump was set on the basis of the terminal half-life being simulated. A series of dosage regimens were designed to generate different CEQ concentration profiles with terminal half-lives of 3 and $6 \mathrm{~h}$ for three consecutive administrations in this apparatus.

\section{In Vitro PK/PD Model and CEQ Dosing Regimens}

The apparatus ran under $37^{\circ} \mathrm{C}$. The elimination terminal halflives of cefquinome reported in the literature varied from 0.5 to $10 \mathrm{~h}$ (Limbert et al., 1991; Li et al., 2008; Al-Taher, 2010; Tohamy, 2011; Yuan et al., 2011; Zonca et al., 2011; Dumka et al., 2013), therefore, 3 and $6 \mathrm{~h}$ were selected in our study. Peristaltic pumps circulated fresh MHB medium to and from the central compartment at a flow rate of $15.75 \mathrm{r} / \mathrm{min}$ for $t_{1 / 2 \beta}$ of $3 \mathrm{~h}$ or $7.86 \mathrm{r} / \mathrm{min}$ for $\mathrm{t}_{1 / 2 \beta}$ of $6 \mathrm{~h}$, respectively. $3 \mathrm{~mL}$ bacterial suspensions $\left(10^{8} \mathrm{CFU} / \mathrm{mL}\right)$ at the logarithmic phase were injected into the central compartment container. The bacteria were incubated in the model for $2 \mathrm{~h}$ to result in exponentially growing cultures before addition of cefquinome. Antibiotic doses were calculated to generate CEQ initial concentrations of $1 / 2 \mathrm{MIC}$, MIC, 2MIC, 4MIC, 8MIC, and 16MIC levels, respectively. The in vitro pharmacokinetics of cefquinome for all doses after three consecutive administrations with $24 \mathrm{~h}$ interval for terminal halflives of 3 and $6 \mathrm{~h}$ were simulated.

\section{Quantification of Cefquinome in MHB}

Samples were collected from the central compartment immediately before and at $0.5,1.0,2.0,3.0,4.0,6.0,8.0$, $10.0,12.0$, and $24.0 \mathrm{~h}$ after the first and second administration, and $0.5,1.0,2.0,3.0,4.0,6.0,8.0,10.0,12.0,24.0,30.0,36.0$, and $48.0 \mathrm{~h}$ after the third administration. These samples were stored at $-20^{\circ} \mathrm{C}$ until analysis.

$1.5 \mathrm{~mL}$ of $\mathrm{MHB}$ was extracted for the determination of cefquinome. The concentrations of cefquinome in MHB were analyzed by high-performance liquid chromatography-MS/MS (HPLC-MS/MS) with an assay range of $0.005-0.5 \mu \mathrm{g} / \mathrm{mL}$. The protein in $\mathrm{MHB}$ sample $(1 \mathrm{~mL})$ was precipitated by $2 \mathrm{~mL}$ acetonitrile, and the supernatant was directly injected into HPLC-MS/MS after high speed centrifugation. Analysis of quality control (QC) samples at three levels (0.01, 0.05, $0.2 \mu \mathrm{g} / \mathrm{mL}$ ) showed that the recoveries of the method were above $70 \%$; the intra-day and inter-day coefficients of variation were within $15 \%$. The pharmacokinetic parameters were calculated by a WinNonlin software (version 5.2.1; Pharsight Corporation, USA).

\section{In Vitro Time-Kill Experiments and Susceptibility Testing of $S$. aureus}

To measure the antimicrobial effect of cefquinome with different terminal half-lives against $S$. aureus, the colony count and susceptibility of bacteria in each time point were performed after treatment. Half of each sample was subjected to time-kill kinetic assays. Samples $(100 \mu \mathrm{L})$ in series of 10 -fold dilution with sterile saline $(0.9 \% \mathrm{NaCl})$ were spread onto Mueller-Hinton agar to determine the number of total or resistant cells. The $\log _{10}$ of surviving $S$. aureus cells $(\mathrm{CFU} / \mathrm{mL})$ was plotted against each time point. The other half of each sample was used for the susceptibility test. MIC values of bacteria post-exposed in each time point was conducted using tube dilution method according to the criteria established by the Clinical Laboratory Standards Institute [CLSI] (2013).

\section{Pharmacokinetic/Pharmacodynamic (PK/PD) Integration Analysis}

By using the individual pharmacokinetic result from each in vitro dosage regimen, the following $\mathrm{PK} / \mathrm{PD}$ parameters were obtained: ratio of area under the curve of cefquinome concentration versus time to $\mathrm{MIC}\left(\mathrm{AUC}_{0-\infty} / \mathrm{MIC}\right)$ or $\left(\mathrm{AUC}_{0-24 \mathrm{~h}} / \mathrm{MIC}\right)$, ratio of area under the curve of cefquinome concentration exceeding $\mathrm{MIC}$ to $\mathrm{MIC}\left(\mathrm{AUC}_{\mathrm{C}}>\mathrm{MIC} / \mathrm{MIC}\right)$, time of concentration above MIC ( $\mathrm{T}>\mathrm{MIC})$ or MPC ( $>$ MPC), time of concentration within MPC and MIC ( $\left.\mathrm{T}_{\text {MSW }}\right)$ or expressed as percentage $\left(\mathrm{T}_{\mathrm{MSW}} \%\right)$, ratio of $\mathrm{MIC}_{72}$ to $\mathrm{MIC}_{\text {initial }}\left(\mathrm{MIC}_{72} / \mathrm{MIC}_{\text {initial }}\right)$.

Gaussian function was used to simulate the correlation of $\mathrm{MIC}_{72 \mathrm{~h}} / \mathrm{MIC}_{\text {initial }}$ with $\mathrm{T}_{\mathrm{MSW}} \%$ (percentage of the time during which cefquinome concentrations were inside the MSW). The formula was as follows:

$$
y=y_{0}+\frac{A}{\sqrt{2 \pi} \sigma} e^{-\frac{\left(x-x_{0}\right)^{2}}{2 \sigma^{2}}}
$$

where $\mathrm{A}$ was the area under curve and upper baseline; $\mathrm{x}_{0}$ was the minimum value of $\mathrm{T}_{\mathrm{MSW}} \%$ which resulted in the maximum $\mathrm{MIC}_{72 \mathrm{~h}} / \mathrm{MIC}_{\text {initial }}$.

\section{RESULTS}

\section{$\mathrm{MIC}_{99}$ and MPC}

The MIC $_{99}$ and MPC of cefquinome against S. aureus ATCC 25923 were 0.4 and $4.096 \mu \mathrm{g} / \mathrm{mL}$, respectively. So the SI was 10.24 (MPC/MIC99).

\section{In Vitro Simulated Pharmacokinetics}

The in vitro simulated time-concentration curves for cefquinome with terminal half-lives of 3 and $6 \mathrm{~h}$ are shown in Figure 2. This study used time-concentration curves of the unbound fractions of cefquinome in Mueller-Hinton broth. The maximum concentrations were approximately equal to $1 / 2 \mathrm{MIC}$ to 16 -fold MIC of cefquinome against S. aureus ATCC 25923 after three 
A

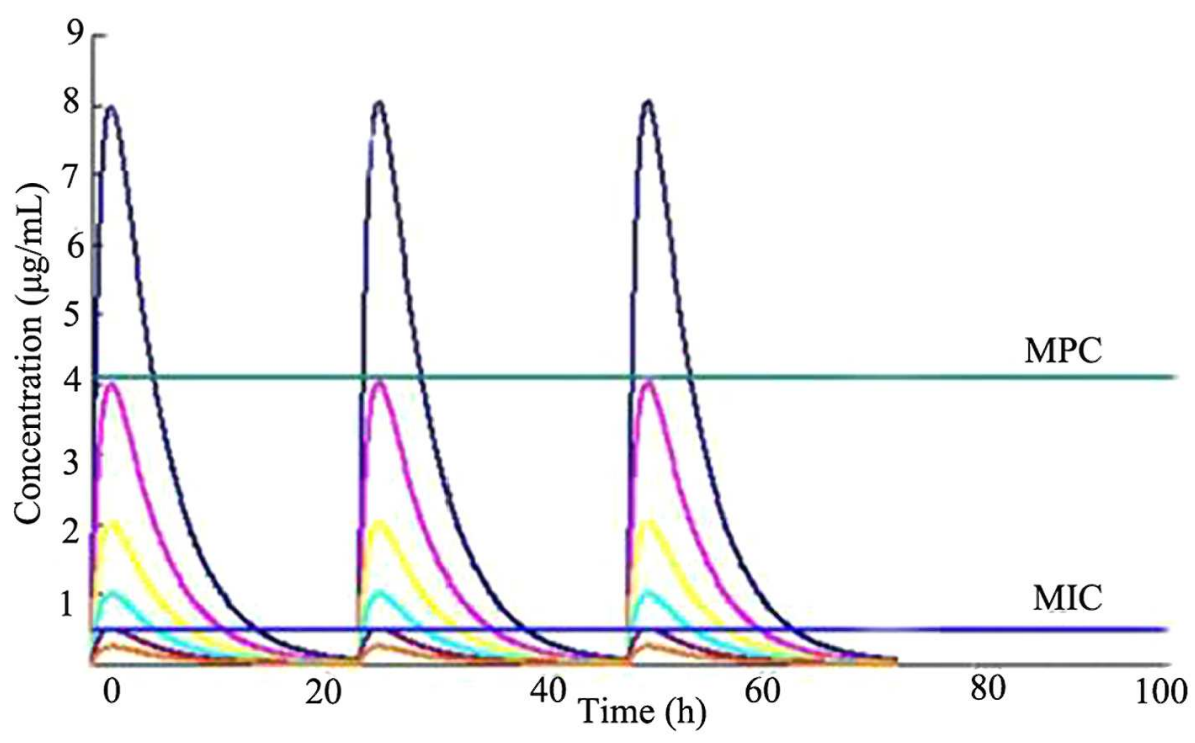

B

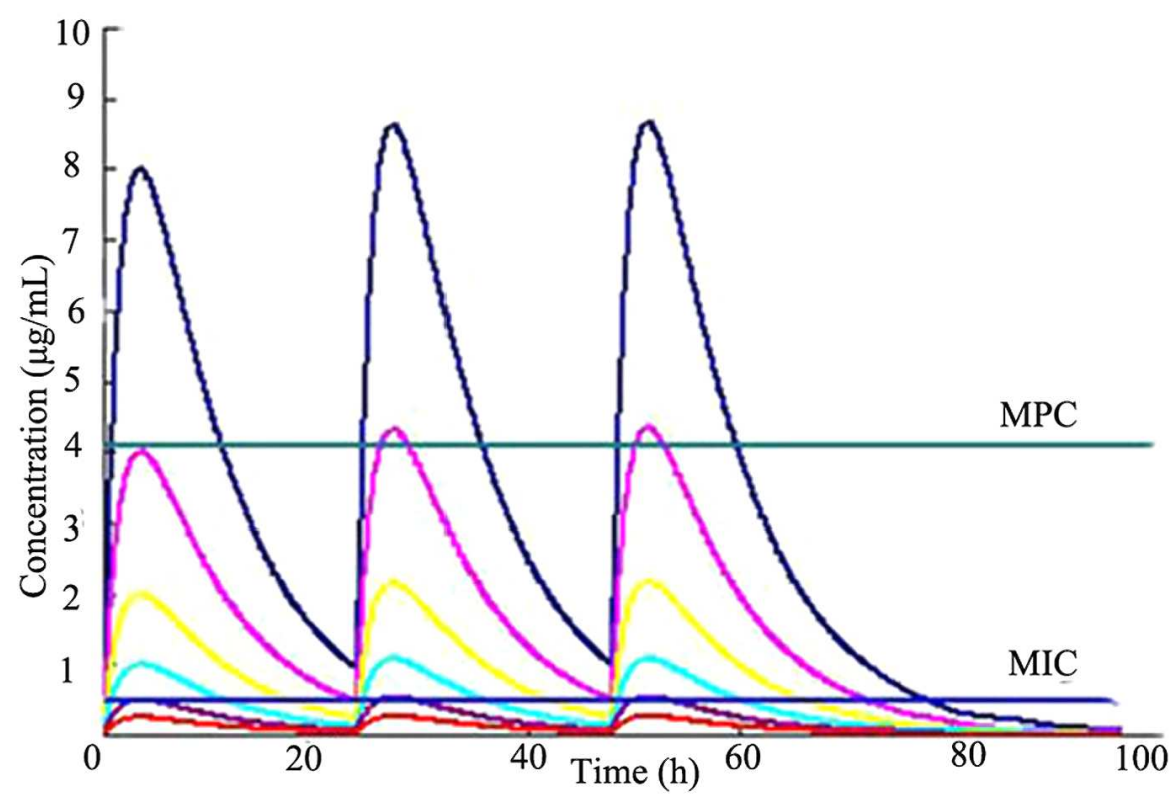

FIGURE 2 | Concentration-time curves of cefquinome in the in vitro pharmacokinetics model of two terminal half-lives ( $\left.t_{1 / 2 \beta}\right)$. The horizontal lines indicate the MPC $(4.096 \mu \mathrm{g} / \mathrm{mL})$ and MIC $(0.4 \mu \mathrm{g} / \mathrm{mL})$ for Staphylococcus aureus ATCC 25923 , respectively. (A) $\mathrm{t}_{1 / 2 \beta}=3 \mathrm{~h}$, (B) $\mathrm{t}_{1 / 2 \beta}=6 \mathrm{~h}$.

consecutive administrations. The MPC and MIC levels were also indicated in the simulated time-concentration curves.

\section{The Bacteria Killing Curves in In Vitro Models}

Time-killing curves of S. aureus ATCC 25923 in the in vitro model under different dosing regimens are shown in the following Figure 3 . For a $t_{1 / 2 \beta}$ of $3 \mathrm{~h}$ multiple dosages, bacteriostatic or bactericidal action was observed when maximum cefquinome concentrations were equal to MIC or over MIC levels (dosages ranged from 0.672 to $10.767 \mathrm{mg}$ ). For a $t_{1 / 2 \beta}$ of $6 \mathrm{~h}$ multiple dosages, continuous bactericidal action was observed when maximum cefquinome concentrations were at $4 \mathrm{MIC}, 8 \mathrm{MIC}, 16 \mathrm{MIC}$ (dose ranged from 2.691 to $10.767 \mathrm{mg}$ ), and the continuous time of bactericidal action at $16 \mathrm{MIC}$ was the longest among three concentrations. 
A

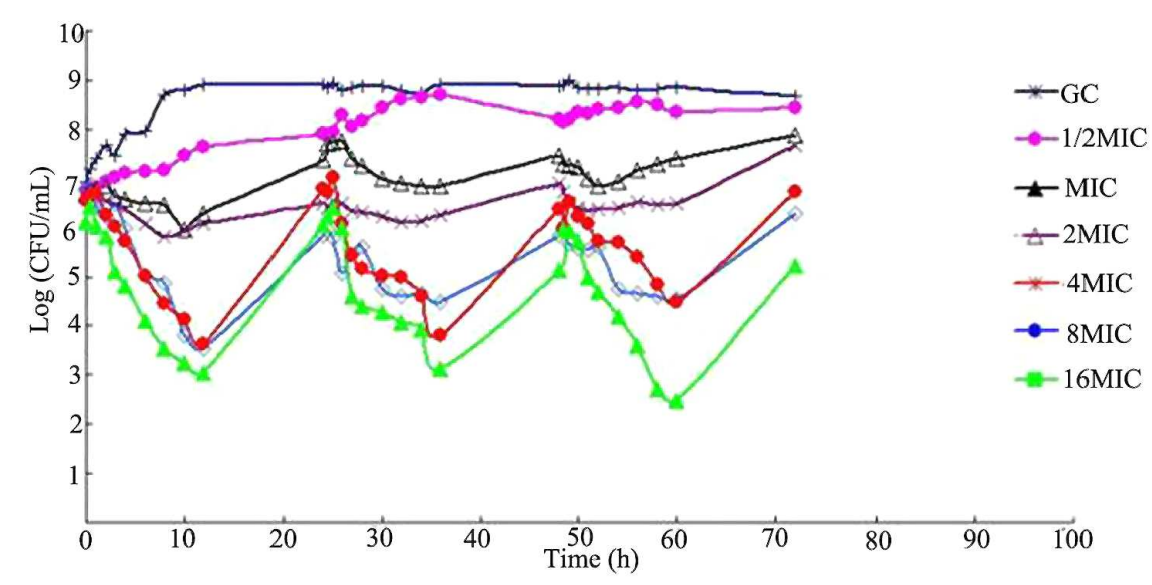

B

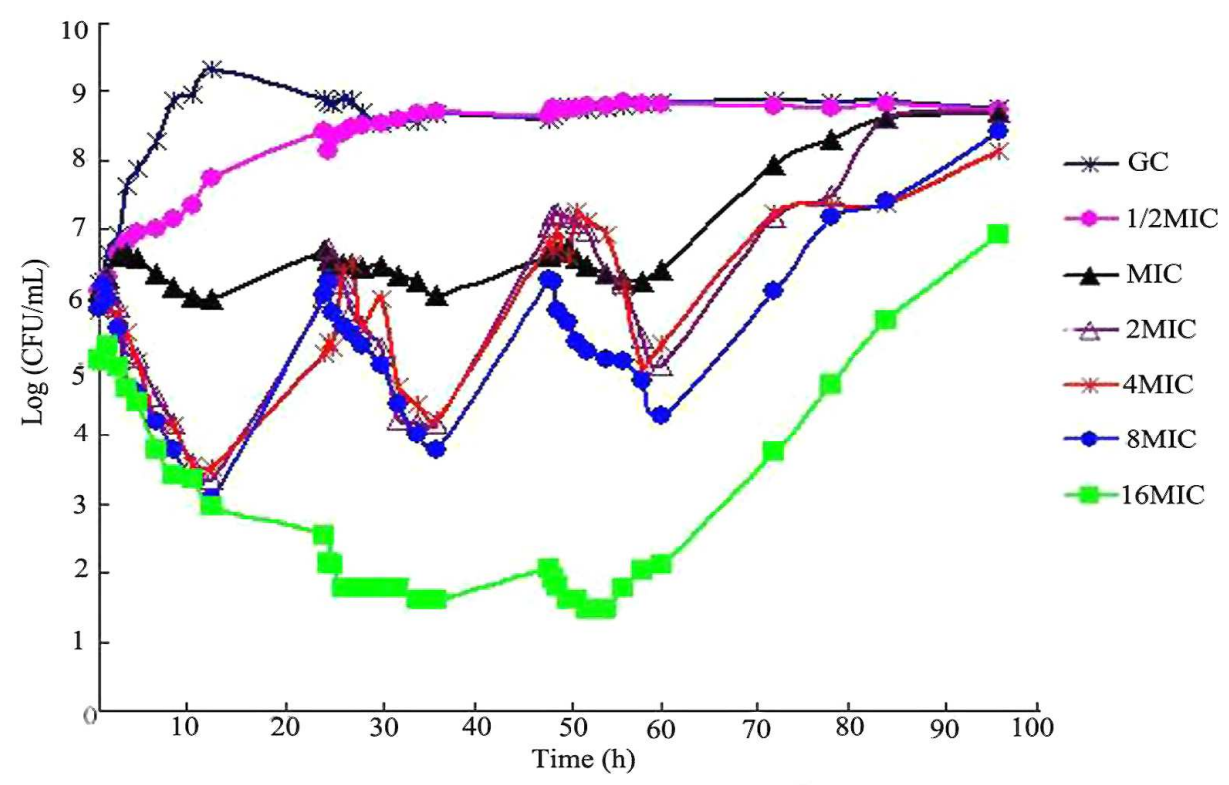

FIGURE 3 | Viable counts of S. aureus ATCCC 25923 in time-kill assays with cefquinome in different terminal half-lives ( $t_{1 / 2}$ ) at cefquinome concentrations from a half to 16 -fold the minimum inhibitory concentration (MIC), in comparison with a cefquinome-free control. (A) $t_{1 / 2} \beta=3 \mathrm{~h}$, (B) $t_{1 / 2 \beta}=6 \mathrm{~h}$.

\section{Loss of Susceptibility to Cefquinome}

Compared to drug-free group, loss of susceptibility was observed in MIC, 2MIC, and 4MIC concentration groups after administrations, as shown in Figure 4. For multiple dosages with $\mathrm{t}_{1 / 2 \beta}$ of $3 \mathrm{~h}$, the bacterial MICs after administration increased to $4.0,8.0$, and $1.0 \mu \mathrm{g} / \mathrm{mL}$ for MIC, $2 \mathrm{MIC}$, and $4 \mathrm{MIC}$ groups, respectively. For multiple dosages with $t_{1 / 2 \beta}$ of $6 \mathrm{~h}$, the bacterial MICs after administration increased to $2.0,4.0$, and $0.8 \mu \mathrm{g} / \mathrm{mL}$ for MIC, and 2MIC, and 4MIC concentration administrations, respectively. The MIC values did not change through the whole experiment period in $8 \mathrm{MIC}$ and 16MIC administration groups with two different terminal half-lives.

\section{Correlation of PK/PD Indices with Resistance Selection}

Pharmacokinetic/pharmacodynamic indices, such as $\mathrm{AUC}_{24} h / \mathrm{MIC}$ (where $\mathrm{AUC}_{24 h}$ is the area under the drug concentration time curve in a $24 \mathrm{~h}$ interval) and time above the MIC, provide an empirical way to relate antimicrobial dose to favor the treatment effect of bactericidal agents. Relationships between $\mathrm{PK} / \mathrm{PD}$ indices and loss of susceptibility are shown in Table 1. For cephalosporin, $\mathrm{T}>\mathrm{MIC}$ was the index most commonly associated with restricting susceptible cell growth. When $\mathrm{T}>$ MIC attained $17.14 \mathrm{~h}$ or $\mathrm{T}>\mathrm{MIC} \%$ attained $24 \%$, the MIC value of cefquinome against $S$. aureus increased to 


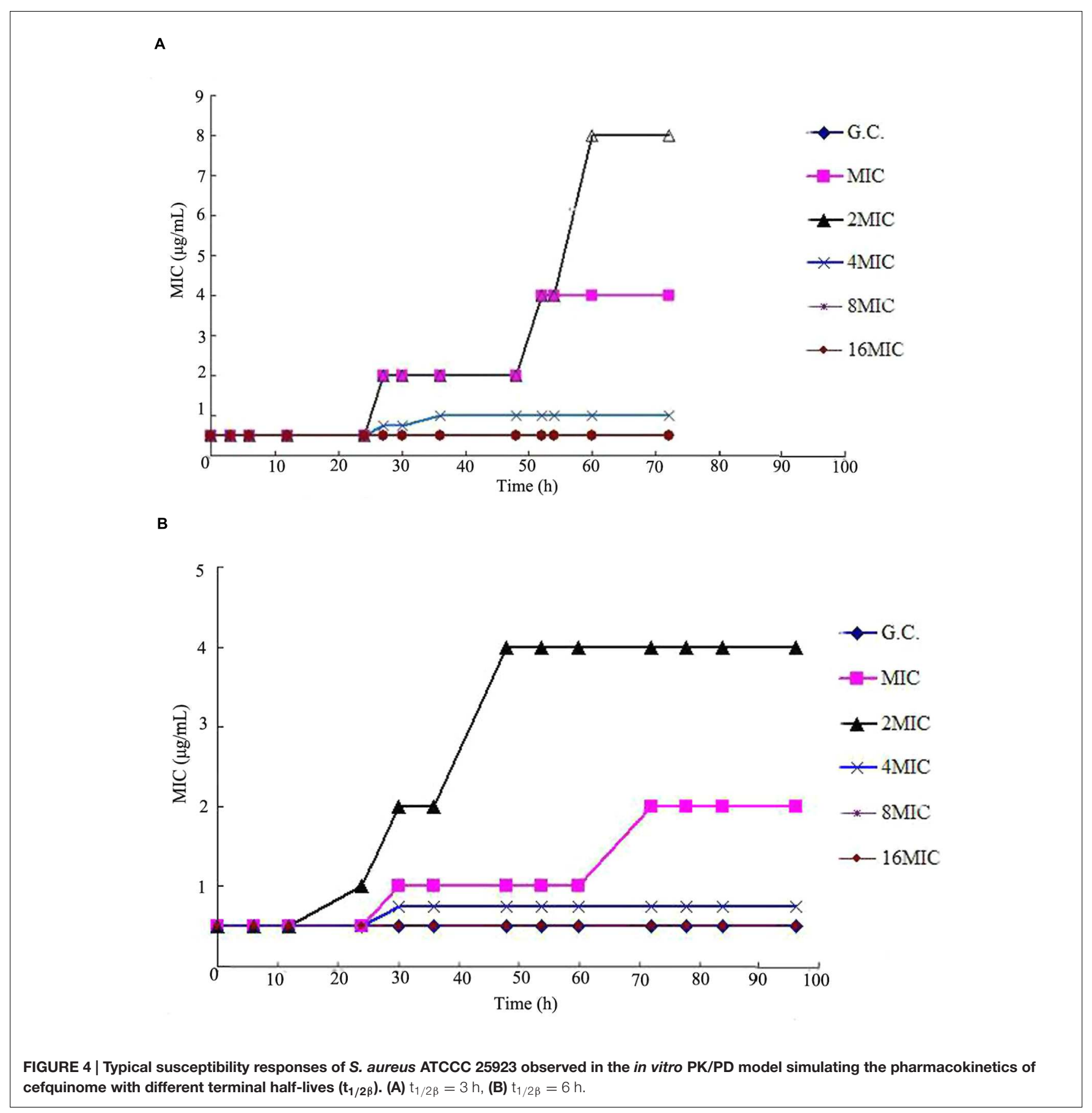

16-fold of initial value at $72 \mathrm{~h}$ after multiple administrations in groups with $t_{1 / 2 \beta}$ of $3 \mathrm{~h}$. Similarly, in groups with $t_{1 / 2 \beta}$ of $6 \mathrm{~h}$, when $\mathrm{T}>\mathrm{MIC}=34.28 \mathrm{~h}$ or $\mathrm{T}>\mathrm{MIC} \%=48 \%$, the value of cefquinome MIC increased to eightfold of initial value after multiple dosages.

Other $\mathrm{PK} / \mathrm{PD}$ indices also showed correlation with the selection of resistance (Table 1). When $\mathrm{T}_{\text {MSW }}$ and $\mathrm{T}_{\text {MSW }} \%$ were $17.14 \mathrm{~h}$ and $24 \%$, the MIC increased to 16 -fold of initial value in groups with $t_{1 / 2 \beta}$ of $3 \mathrm{~h}$ after multiple dosages, and the MIC increased to eightfold of initial value When $\mathrm{T}_{\mathrm{MSW}}$ and $\mathrm{T}_{\mathrm{MSW}} \%$ were $34.28 \mathrm{~h}$ and $48 \%$ with $\mathrm{t}_{1 / 2 \beta}$ of $6 \mathrm{~h}$ after multiple dosages, respectively.

\section{Correlation Analysis of MIC Increase with $\mathrm{T}_{\mathrm{MSW}} \%$}

According to Gaussian function and regression analysis results, the bacteria was prone to develop resistance when $\mathrm{T}_{\mathrm{MSW}} \%$ was about $20 \%\left(\mathrm{x}_{0}=0.2027\right)$ in groups with $\mathrm{t}_{1 / 2 \beta}$ of $3 \mathrm{~h}$ after multiple dosages $\left(R^{2}=0.9989\right)$, and $\mathrm{T}_{\mathrm{MSW}} \%$ was 
TABLE 1 | Pharmacokinetic/pharmacodynamic (PK/PD) indices of antimicrobial efficacy and risk of resistance selection amongst Staphylococcus aureus ATCC 25923 over the complete dosing interval following PK simulations of three consecutive administrations of cefquinome in $t_{1 / 2} \beta$ of 3 and 6 h.

\begin{tabular}{|c|c|c|c|c|c|c|c|c|c|}
\hline$t_{1 / 2 \beta}(h)$ & D (mg) & $\frac{A U C_{C>M I C}}{M I C}$ (h) & $\frac{\mathrm{AUC}_{24}}{M I C}$ (h) & $\mathrm{T}>\mathrm{MIC}(\mathrm{h})$ & $\mathrm{T}>\mathrm{MPC}(\mathrm{h})$ & $\mathbf{T}_{\text {MSW }}(\mathbf{h})$ & $\mathbf{T}_{\mathbf{M S W}} \%$ & $\mathbf{T}>\mathbf{M I C} \%$ & $\frac{\text { MIC }_{72}}{\text { MIC }_{\text {initial }}}$ \\
\hline \multirow[t]{6}{*}{3} & 1/2MIC & 0 & 3.22 & 0 & 0 & 0 & 0 & 0 & 1 \\
\hline & MIC & 6.41 & 6.43 & 8.11 & 0 & 8.11 & 11 & 11 & 8 \\
\hline & $2 \mathrm{MIC}$ & 25.89 & 12.89 & 17.14 & 0 & 17.14 & 24 & 24 & 16 \\
\hline & $4 \mathrm{MIC}$ & 64.68 & 25.76 & 26.13 & 0 & 26.13 & 36 & 36 & 2 \\
\hline & $8 \mathrm{MIC}$ & 142.34 & 51.52 & 35.13 & 7.81 & 27.32 & 38 & 49 & 1 \\
\hline & 16MIC & 297.75 & 103.07 & 44.14 & 16.82 & 27.32 & 38 & 61 & 1 \\
\hline \multirow[t]{6}{*}{6} & 1/2MIC & 0 & 5.96 & 0 & 0 & 0 & 0 & 0 & 1 \\
\hline & MIC & 12.81 & 11.92 & 16.21 & 0 & 16.21 & 23 & 23 & 4 \\
\hline & $2 \mathrm{MIC}$ & 51.77 & 23.89 & 34.28 & 0 & 34.28 & 48 & 48 & 8 \\
\hline & $4 \mathrm{MIC}$ & 129.35 & 47.73 & 52.26 & 0 & 52.26 & 73 & 73 & 1.5 \\
\hline & $8 \mathrm{MIC}$ & 284.68 & 95.45 & 70.26 & 15.63 & 54.64 & 76 & 98 & 1 \\
\hline & 16MIC & 595.50 & 190.96 & 88.28 & 33.64 & 54.64 & 76 & 123 & 1 \\
\hline
\end{tabular}

about $40 \%\left(\mathrm{x}_{0}=0.4102\right)$ in groups with $\mathrm{t}_{1 / 2 \beta}$ of $6 \mathrm{~h}$ after multiple dosages $\left(R^{2}=0.9986\right)$, respectively, as shown in Figure 5. Those results were consistent with the data from Table 1.

\section{DISCUSSION}

Antimicrobial resistance has been a global problem and a great number of strategies have been proposed to slow the emergence of resistance (Stratton, 2003; Levy and Marshall, 2004). In vivo or in vitro $\mathrm{PK} / \mathrm{PD}$ models have previously been applied to optimize the dosage regimen of various antibiotics in the literature (Croisier et al., 2004b; Ferran et al., 2009; Andraud et al., 2011; Gebru et al., 2012). However, PK/PD model has been renewably used to investigate relationship of bacterial resistance with AUC/MIC, $\mathrm{C}_{\max } / \mathrm{MIC}$ and $\mathrm{T}>\mathrm{MIC}$, recently. In the present study, we simulated the cefquinome pharmacokinetic profiles with different terminal half-lives using this dynamic model and tried to predict the selection of resistant $S$. aureus with some indices, such as $\mathrm{T}>\mathrm{MIC}$ and $\mathrm{T}_{\mathrm{MSW}} \%$.

It is believed that integration of drug pharmacokinetics, mutant prevention concentration is helpful in slowing the emergence of resistance. In this experiment, the MPC and MSW of cefquinome against $S$. aureus ATCC25923 were determined. The SI, ratio of MPC to $\mathrm{MIC}_{99}$, is the ability of a drug to select resistant mutants (Zhao and Drlica, 2001). In other word, the bigger the SI is, the easier the drug is to induce resistance. The SI of cefquinome against S. aureus ATCC25923 was 10.24 in our study, which suggested that cefquinome could induce $S$. aureus resistant mutation easily and effectively.

$\mathrm{T}>\mathrm{MIC}$ was regarded as a promising predictor for the efficacy of cephalosporins. Previous pharmacodynamic studies have addressed separate issues around the relationships between $\mathrm{T}>$ MIC and efficacy for cephalosporins. Craig (1995) has reviewed the data of efficacy for cephalosporins against Enterbacteriaceae, streptococci and S. aureus in several animal infection models and found that the time above MIC required for a bacteriostatic effect against strains of Enterbacteriaceae,
Streptococci were generally $60-70 \%$, and $40-50 \%$ for S. aureus, respectively. In our study, the value of $\mathrm{T}>\mathrm{MIC}$ for maximal killing was $40 \sim 60 \%$ for cefquinome against $S$. aureus reference strain. In consistent with the widespread idea that antibacterial efficacy of $\beta$-lactams depend on $\mathrm{T}>\mathrm{MIC}$, the effect of cefquinome against $S$. aureus in our study exhibited similar correlation. As observed in Figure 3, killing curves of S. aureus exposed to each dose of cefquinome showed the typical pattern of time-dependent bactericidal action. The similar observation was also reported for other $\beta$-lactam drugs in previous studies (Lindecrona et al., 2000; Burgess and Hall, 2004). Considering that the $\mathrm{T}>\mathrm{MIC}$ was one of the most important parameters for optimal dosage regimen, $\mathrm{T}>\mathrm{MIC}$ was selected as a parameter to evaluate resistant mutation in this article. In the present study, the values of $\mathrm{T}>\mathrm{MIC}$ ranging from 8.11 to $17.14 \mathrm{~h}$ were dangerous zones for inducing resistant mutation in groups with $t_{1 / 2 \beta}$ of $3 \mathrm{~h}$ after multiple dosages, and values ranging from 16.21 to $34.28 \mathrm{~h}$ were dangerous zones for inducing resistant mutation in groups with $t_{1 / 2 \beta}$ of $6 \mathrm{~h}$ multiple dosages. Compared these values with previously reported study data that $\mathrm{T}>\mathrm{MIC}$ value ranged from 42 to $54 \mathrm{~h}$ for cefquinome in dairy cows (Zonca et al., 2011), the prevention of selecting resistant $S$. aureus strains seems to be achieved by the present dosage regimens approved by the European Medicines Agency (EMA) for the treatment of dairy cow mastitis.

$\mathrm{T}_{\text {MSW }}$ is another important parameter used for evaluating resistant mutation. A previous study conducted in a rabbit lung infection model with Streptococcus pneumonia showed that the selection of resistant bacteria occurred systematically when concentrations of gatifloxacin were within the MSW ( $\mathrm{T}_{\mathrm{MSW}}$ ) for more than $45 \%$ of the treatment duration (Croisier et al., 2004b). Another experiment in rabbits infected by S. aureus also showed that time in the MSW > 33\% was preferable to select mutants (Cui et al., 2006). When the abilities of the indices $\mathrm{T}_{\mathrm{MSW}}, \mathrm{AUC}_{24} \mathrm{~h} / \mathrm{MIC}, \mathrm{C}_{\max } / \mathrm{MIC}$ to predict the selection of resistant bacteria were compared, only $\mathrm{T}_{\mathrm{MSW}}$ appeared to be a good predictor of the prevention of resistance (Ferran et al., 2009). In this study, $T_{\text {MSW }}$ was also used to predict selection of 
A

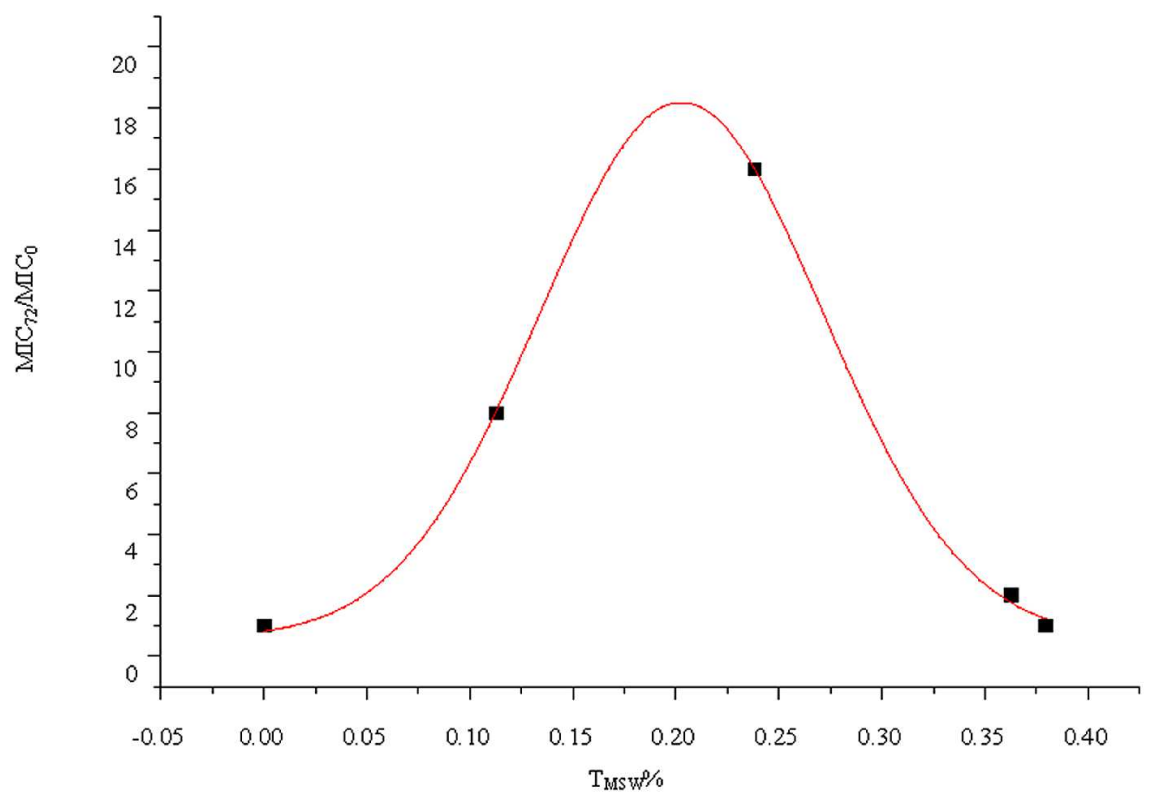

B

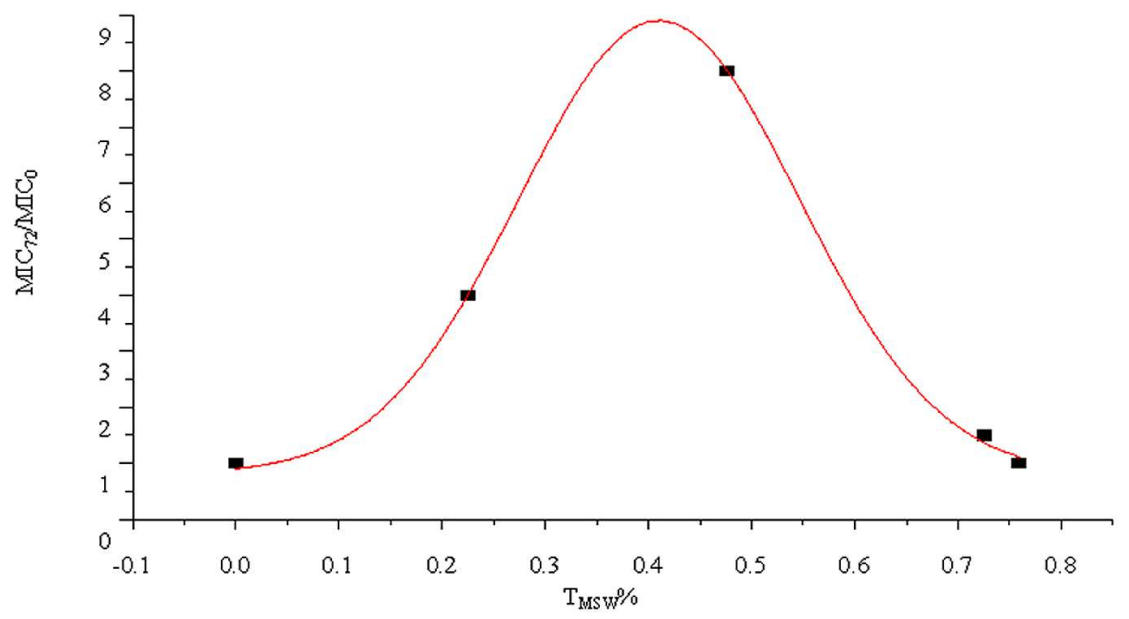

FIGURE 5 | Relationship between the $\mathrm{T}_{\mathrm{MSw}} \%$ and $\mathrm{MIC}_{72} / \mathrm{MIC}_{0}$ for S. aureus ATCC 25293 exposed to cefquinome in the in vitro model with two terminal half-lives $\left(\mathbf{t}_{1 / 2 \beta}\right)$. (A) $t_{1 / 2 \beta}=3 h$, (B) $t_{1 / 2 \beta}=6 \mathrm{~h}$.

resistant $S$. aureus against cefquinome. It was clearly determined that when $\mathrm{T}_{\mathrm{MSW}}$ was above $36 \%$ in groups with $\mathrm{t}_{1 / 2 \beta}$ of $3 \mathrm{~h}$ after multiple dosages or above $73 \%$ in groups with $\mathrm{t}_{1 / 2 \beta}$ of $6 \mathrm{~h}$ after multiple dosages, cefquinome could restrict the resistant mutation.

In the present investigation, the bacterial resistant mutation was affected by different terminal half-lives and dosages. Within the group of the same terminal half-life ( 3 or $6 \mathrm{~h}$ ), the bacterial resistant mutation happened under the condition of relative low dosage (maximum concentrations were 2MIC and 4MIC). When the maximum concentration was over $8 \mathrm{MIC}$, resistant mutation was prevented. In the same dosages (maximum concentration was $2 \mathrm{MIC}$ or $4 \mathrm{MIC}$ ), the $\mathrm{MIC}_{72} h$ increased from 8 - to 16 -fold in groups with $\mathrm{t}_{1 / 2 \beta}$ of $3 \mathrm{~h}$ after multiple dosages and increased from 4- to 8 -fold in groups with $\mathrm{t}_{1 / 2 \beta}$ of $6 \mathrm{~h}$ after multiple dosages, respectively. Those results suggested that the bacterial resistant mutation might happen at a low concentration. In high dosages or long terminal half-lives, the drug concentrations might exceed the MPC value, the upper boundary of MSW, and the resistant mutation could be inhibited. The conclusion from this study was consistent with other similar studies (Cui et al., 2006; Ferran et al., 2009; Zhu et al., 2012). 
The limitation of the study was that only one reference strain was used to simulate the efficacy of cefquinome in this in vitro model; however, the multi-resistance is becoming an urgent trend all over the world. It may be reasonable that more clinical isolates of $S$. aureus could be used for further study. Firsov et al. (2008) used two methicillinresistant strains of S. aureus, ATCC 6538 and ATCC 43300, to study the enrichment of ciprofloxacin resistant mutation in an in vitro dynamic model. Liang et al. (2011), chose three clinical isolates of $S$. aureus, SA99, RN450 and RN450-A1, to analyze the enrichment of levofloxacin resistant mutation with in vitro dynamic model. From the results obtained in the dynamic model, more clinical isolates could be used to study resistant mutation easily based on the substantial method. Moreover, to our knowledge, evaluation of antimicrobial activity for cefquinome with different terminal half-lives is scarce. Considering the fact that cefquinome is the time-dependent drug, it is positive to study the correlation resistant mutation with different terminal half-life conditions. Another limitation of the current study was the absence of resistance mechanisms of $S$. aureus observed in vitro. Therefore, the pattern of $S$. aureus resistant to cefquinome, such as production of $\beta$-lactamases, alteration of drug targets, porin-mediated resistance, and/or efflux-mediated resistance, needs confirmation in the further study.

\section{REFERENCES}

Allen, G. P., and Hankins, C. D. (2009). Evaluation of the mutant selection window for fluoroquinolones against Neisseria gonorrhoeae. J. Antimicrob. Chemother. 64, 359-363. doi: 10.1093/jac/dkp172

Allen, G. P., Kaatz, G. W., and Rybak, M. J. (2004). In vitro activities of mutant prevention concentration-targeted concentrations of fluoroquinolones against Staphylococcus aureus in a pharmacodynamic model. Int. J. Antimicrob. Agents 24, 150-160. doi: 10.1016/j.ijantimicag.2004. 03.011

Al-Taher, A. (2010). Pharmacokinetics of cefquinome in camels. J. Animal Vet. Adv. 9, 848-852. doi: 10.3923/javaa.2010.848.852

Andraud, M., Chauvin, C., Sanders, P., and Laurentie, M. (2011). Pharmacodynamic modeling of in vitro activity of marbofloxacin against Escherichia coli strains. Antimicrob. Agents Chemother. 55, 756-761. doi: 10.1128/AAC.00865-10

Archer, G. L. (1998). Staphylococcus aureus: a well-armed pathogen. Clin. Infect. Dis. 26, 1179-1181. doi: 10.1086/520289

Azizoglu, R. O., Lyman, R., and Anderson, K. L. (2013). Bovine Staphylococcus aureus: dose response to iodine and chlorhexidine and effect of iodine challenge on antibiotic susceptibility. J. Dairy Sci. 96, 993-999. doi: 10.3168/jds. 2012-5857

Blondeau, J., Borsos, S., Blondeau, L., Blondeau, B., and Hesje, C. (2012). Comparative minimum inhibitory and mutant prevention drug concentrations of enrofloxacin, ceftiofur, florfenicol, tilmicosin and tulathromycin against bovine clinical isolates of Mannheimia haemolytica. Vet. Microbiol. 160, 85-90. doi: 10.1016/j.vetmic.2012.05.006

Bronner, S., Murbach, V., Peter, J.-D., Levêque, D., Elkhaili, H., Salmon, Y., et al. (2002). Ex vivo pharmacodynamics of amoxicillin-clavulanate against $\beta$-lactamase-producing Escherichia coli in a Yucatan miniature pig model that mimics human pharmacokinetics. Antimicrob. Agents Chemother. 46, 37823789. doi: 10.1128/AAC.46.12.3782-3789.2002

Burgess, D. S., and Hall, R. G. II, (2004). In vitro killing of parenteral beta-lactams against standard and high inocula of extended-spectrum beta-lactamase and non-esbl producing Klebsiella pneumoniae. Diagn. Microbiol. Infect. Dis. 49, 41-46. doi: 10.1016/j.diagmicrobio.2003.11.007

\section{CONCLUSION}

The present study of resistant mutation with $S$ aureus ATCC 25923 exposed to cefquinome with two different terminal halflives in in vitro dynamic models supports the MSW hypothesis and provides some useful parameters to predict the resistant mutation, especially $\mathrm{T}>\mathrm{MIC}$ and $\mathrm{T}_{\mathrm{MSW}} \%$. Further study will concentrate on more clinical isolates which could be used for verification of these results and resistance patterns of $S$. aureus resistant to cefquinome.

\section{AUTHOR CONTRIBUTIONS}

All authors listed, have made substantial, direct and intellectual contribution to the work, and approved it for publication.

\section{ACKNOWLEDGMENTS}

The authors would like to thank the financial support by the Natural Science Foundation of China (grant number 31172366) and 973 program (grant number 2013CB127200/ 2013CB127203).

Cavaco, L., Abatih, E., Aarestrup, F. M., and Guardabassi, L. (2008). Selection and persistence of CTX-M-producing Escherichia coli in the intestinal flora of pigs treated with amoxicillin, ceftiofur, or cefquinome. Antimicrob. Agents Chemother. 52, 3612-3616. doi: 10.1128/AAC.00354-08

Clinical Laboratory Standards Institute (CLSI) (2013). Performance Standards for Antimicrobial Disk, and Dilution Susceptibility Tests for Bacteria Isolated From Animals, Approved Standard-Fourth Edn. Wayne, PA: Clinical Laboratory Standards Institute.

Craig, W. A. (1995). Interrelationship between pharmacokinetics and pharmacodynamics in determining dosage regimens for broadspectrum cephalosporins. Diagn. Microbiol. Infect. Dis. 22, 89-96. doi: 10.1016/0732-8893(95)00053-D

Croisier, D., Etienne, M., Bergoin, E., Charles, P.-E., Lequeu, C., Piroth, L., et al. (2004a). Mutant selection window in levofloxacin and moxifloxacin treatments of experimental pneumococcal pneumonia in a rabbit model of human therapy. Antimicrob. Agents Chemother. 48, 1699-1707. doi: 10.1128/AAC.48.5.16991707.2004

Croisier, D., Etienne, M., Piroth, L., Bergoin, E., Lequeu, C., Portier, H., et al. (2004b). In vivo pharmacodynamic efficacy of gatifloxacin against Streptococcus pneumoniae in an experimental model of pneumonia: impact of the low levels of fluoroquinolone resistance on the enrichment of resistant mutants. J. Antimicrob. Chemother. 54, 640-647. doi: 10.1093/jac/dkh393

Cui, J., Liu, Y., Wang, R., Tong, W., Drlica, K., and Zhao, X. (2006). The mutant selection window in rabbits infected with Staphylococcus aureus. J. Infect. Dis. 194, 1601-1608. doi: 10.1086/508752

Dong, Y., Zhao, X., Domagala, J., and Drlica, K. (1999). Effect of fluoroquinolone concentration on selection of resistant mutants of Mycobacterium bovis BCG and Staphylococcus aureus. Antimicrob. Agents Chemother. 43, 1756-1758.

Dumka, V. K., Dinakaran, V., Ranjan, B., and Rampal, S. (2013). Comparative pharmacokinetics of cefquinome following intravenous and intramuscular administration in goats. Small Ruminant Res. 113, 273-277. doi: 10.1016/j.smallrumres.2013.02.010

Ferran, A., Dupouy, V., Toutain, P.-L., and Bousquet-Mélou, A. (2007). Influence of inoculum size on the selection of resistant mutants of Escherichia coli in relation to mutant prevention concentrations of marbofloxacin. Antimicrob. Agents Chemother. 51, 4163-4166. doi: 10.1128/AAC.00156-07 
Ferran, A. A., Kesteman, A.-S., Toutain, P.-L., and Bousquet-Mélou, A. (2009). Pharmacokinetic/pharmacodynamic analysis of the influence of inoculum size on the selection of resistance in Escherichia coli by a quinolone in a mouse thigh bacterial infection model. Antimicrob. Agents Chemother. 53, 3384-3390. doi: 10.1128/AAC.01347-08

Firsov, A. A., Lubenko, I. Y., Smirnova, M. V., Strukova, E. N., and Zinner, S. H. (2008). Enrichment of fluoroquinolone-resistant Staphylococcus aureus: oscillating ciprofloxacin concentrations simulated at the upper and lower portions of the mutant selection window. Antimicrob. Agents Chemother. 52, 1924-1928. doi: 10.1128/AAC.01371-07

Gebru, E., Damte, D., Choi, M.-J., Lee, S.-J., Kim, Y.-H., and Park, S. C. (2012). Mutant prevention concentration and phenotypic and molecular basis of fluoroquinolone resistance in clinical isolates and in vitro-selected mutants of Escherichia coli from dogs. Vet. Microbiol. 154, 384-394. doi: 10.1016/j.vetmic.2011.07.033

Grasso, S., Meinardi, G., De Carneri, I., and Tamassia, V. (1978). New in vitro model to study the effect of antibiotic concentration and rate of elimination on antibacterial activity. Antimicrob. Agents Chemother. 13, 570-576. doi: 10.1128/AAC.13.4.570

Guardabassi, L., Schwarz, S., and Lloyd, D. H. (2004). Pet animals as reservoirs of antimicrobial-resistant bacteria review. J. Antimicrob. Chemother. 54, 321-332. doi: $10.1093 / \mathrm{jac} / \mathrm{dkh} 332$

Harris, S. R., Feil, E. J., Holden, M. T., Quail, M. A., Nickerson, E. K., Chantratita, N., et al. (2010). Evolution of MRSA during hospital transmission and intercontinental spread. Science 327, 469-474. doi: 10.1126/science. 1182395

Le Loir, Y., Baron, F., and Gautier, M. (2003). Staphylococcus aureus and food poisoning. Genet. Mol. Res. 2, 63-76.

Levy, S. B., and Marshall, B. (2004). Antibacterial resistance worldwide: causes, challenges and responses. Nat. Med. 10, S122-S129. doi: 10.1038/nm1145

Li, X., Wu, W., Su, D., Wang, Z., Jiang, H., and Shen, J. (2008). Pharmacokinetics and bioavailability of cefquinome in healthy piglets. J. Vet. Pharmacol. Ther. 31, 523-527. doi: 10.1111/j.1365-2885.2008.00989.x

Liang, B., Bai, N., Cai, Y., Wang, R., Drlica, K., and Zhao, X. (2011). Mutant prevention concentration-based pharmacokinetic/pharmacodynamic indices as dosing targets for suppressing the enrichment of levofloxacin-resistant subpopulations of Staphylococcus aureus. Antimicrob. Agents Chemother. 55, 2409-2412. doi: 10.1128/AAC.00975-10

Limbert, M., Isert, D., Klesel, N., Markus, A., Seeger, K., Seibert, G., et al. (1991). Antibacterial activities in vitro and in vivo and pharmacokinetics of cefquinome (HR 111V), a new broad-spectrum cephalosporin. Antimicrob. Agents Chemother. 35, 14-19. doi: 10.1128/AAC. 35.1 .14

Lindecrona, R., Friis, C., and Aarestrup, F. M. (2000). The pharmacodynamic effect of amoxicillin and danofloxacin against Salmonella typhimurium in an in-vitro pharmacodynamic model. Res. Vet. Sci. 68, 261-264. doi: 10.1053/rvsc. 2000.0374

Lowy, F. D. (1998). Staphylococcus aureus infections. New Engl. J. Med. 339, 520-532. doi: 10.1056/NEJM199808203390806

Mohammed Fayaz, A., Girilal, M., Mahdy, S. A., Somsundar, S., Venkatesan, R., and Kalaichelvan, P. (2011). Vancomycin bound biogenic gold nanoparticles: a different perspective for development of anti VRSA agents. Process Biochem. 46, 636-641. doi: 10.1016/j.procbio.2010.11.001

Stratton, C. W. (2003). Dead bugs don't mutate: susceptibility issues in the emergence of bacterial resistance. Emerg. Infect. Dis. 9:10. doi: 10.3201/ eid0901.020172

Tauxe, R. V. (2002). Emerging foodborne pathogens. Int. J. Food Microbiol. 78, 31-41. doi: 10.1016/S0168-1605(02)00232-5

Tohamy, M. (2011). Age-related intramuscular pharmacokinetics of cefquinome in sheep. Small Ruminant Res. 99, 72-76. doi: 10.1016/j.smallrumres.2011.03.004

Wang, L., Yuanshu, Z., Yuhan, Z., and Yingxia, L. (2010). Mutant prevention concentrations of fluoroquinolones against Campylobacter jejuni isolated from chicken. Vet. Microbiol. 144, 409-414. doi: 10.1016/j.vetmic.2010.02.020

Yuan, L., Sun, J., Wang, R., Sun, L., Zhu, L., Luo, X., et al. (2011). Pharmacokinetics and bioavailability of cefquinome in healthy ducks. Am. J. Vet. Res. 72, 122-126. doi: 10.2460/ajvr.72.1.122

Zhao, X., and Drlica, K. (2001). Restricting the selection of antibiotic-resistant mutants: a general strategy derived from fluoroquinolone studies. Clin. Infect. Dis. 33, S147-S156. doi: 10.1086/321841

Zhao, X., and Drlica, K. (2002). Restricting the selection of antibiotic-resistant mutant bacteria: measurement and potential use of the mutant selection window. J. Infect. Dis. 185, 561-565. doi: 10.1086/338571

Zhu, Y.-L., Hu, L.-F., Mei, Q., Cheng, J., Liu, Y.-Y., Ye, Y., et al. (2012). Testing the mutant selection window in rabbits infected with methicillin-resistant Staphylococcus aureus exposed to vancomycin. J. Antimicrob. Chemother. 67, 2700-2706. doi: 10.1093/jac/dks280

Zonca, A., Gallo, M., Locatelli, C., Carli, S., Moroni, P., Villa, R., et al. (2011). Cefquinome sulfate behavior after intramammary administration in healthy and infected cows. J. Dairy Sci. 94, 3455-3461. doi: 10.3168/jds.2010-4109

Conflict of Interest Statement: The authors declare that the research was conducted in the absence of any commercial or financial relationships that could be construed as a potential conflict of interest.

Copyright (c) 2016 Li, Feng, Gu, Yang, Zeng, Zhang and Ding. This is an open-access article distributed under the terms of the Creative Commons Attribution License (CC BY). The use, distribution or reproduction in other forums is permitted, provided the original author(s) or licensor are credited and that the original publication in this journal is cited, in accordance with accepted academic practice. No use, distribution or reproduction is permitted which does not comply with these terms. 